\title{
REFERENCES
}

1. G. H. Hardy and J. E. Littlewood, A further note on the converse of Abel's theorem, Proc. London Math. Soc. 25 (1926), 219-236.

2. A. E. Ingham, On the "high-indices" theorem of Hardy and Littlewood, Quart. J. Math. 8 (1937), 1-7.

3. A. Zygmund, On certain integrals, Trans. Amer. Math. Soc. 55 (1944), 170-204.

UNIVERSITY OF WISCONSIN

\section{REGULAR BOUNDARY POINTS IN MARKOV CHAINS}

\section{A. W. KNAPP 1}

1. Introduction. In classical potential theory [4], a boundary point $p$ of a bounded domain $D$ in Euclidean space is called regular if there is a continuous superharmonic function $h \geqq 0$ defined in $D$ which tends to zero at $p$ and which has a strictly positive lower bound outside any neighborhood of $p$. The tip of a Lebesgue spine is an example of a point which is not regular. The theorem is that the Dirichlet problem for $D$ is solvable for continuous boundary values if and only if every point of the boundary is regular. Moreover, the natural candidate for a solution approaches the given boundary values at every regular point.

The question arises as to the nature of the set of regular boundary points of a domain. It is known [2, p. 225] that this set is not empty and, in fact, that its complement in the boundary has exterior capacity zero.

The same question can be and has been asked for Markov chains. If $P$ is the transition matrix of a transient Markov chain, then a function (column vector) $h$ is harmonic if it has the mean value property $h=P h$, superharmonic if $h \geqq P h$. Doob [1] and Hunt [3] have developed a Martin boundary theory for such a chain, and they showed that, in terms of the space they construct, there is the usual sort of Poisson-integral representation theorem for the integrable nonnegative superharmonic functions. In this framework we ask two questions: Is the set of regular points a Borel set? Is the set neces-

Received by the editors August 2, 1965.

1 This research was supported by Air Force Office of Scientific Research Grant AF-AFOSR 335-63. 
sarily nonempty? The answers are yes to the first and no to the second.

2. Notation and boundary theory. We use the notation of [5]. $P$ is the transition matrix for a countable-state discrete-time Markov chain with only transient states and with starting vector $\pi$, state space $S$, and measure $\operatorname{Pr}_{\pi}$. We do not assume that $P$ has row sums one. We let $P r_{i}$ be the measure for the process when $\pi$ is replaced by a unit mass at $i$, and we let $N=\sum_{n=0}^{\infty} P^{n}$. We shall assume that $\pi N$ is strictly positive.

The Martin boundary for $(\pi, P)$ is constructed as follows. If

$$
K(i, j)=\frac{N(i, j)}{(\pi N)(j)}
$$

then a metric $\rho$ is defined on $S$ by

$$
\rho\left(j, j^{\prime}\right)=\sum_{i} w_{i}(\pi N)(i)\left|K(i, j)-K\left(i, j^{\prime}\right)\right|,
$$

where the weights $w_{i}$ are positive weights with $\sum w_{i} N(i, i)<\infty$. The Cauchy completion of $S$ is a compact metric space $S^{*}$, and $K(i, \cdot)$ and $\rho(\cdot, \cdot)$ extend to be continuous on $S^{*}$ and $S^{*} \times S^{*}$. A sequence $\left\{x_{n}\right\}$ converges to $x$ in $S^{*}$ if and only if $\lim K\left(i, x_{n}\right)=K(i, x)$ for every $i$. The set $S^{*}-S$ is the Martin boundary. On almost every sample path $\omega$ either the path terminates in finite time at a state $x_{v}(\omega)$ or the states on the path converge in $S^{*}$ to a limit $x_{v}(\omega)$.

The Borel probability measure $\mu$ defined on $S^{*}$ by $\mu(E)=P r_{\pi}\left[x_{v} \in E\right]$ is called harmonic measure. It satisfies

$$
\operatorname{Pr}_{i}\left[x_{v} \in E\right]=\int_{S^{*}} K(i, x) d \mu(x) .
$$

Every superharmonic function $h \geqq 0$ with $\pi h<\infty$ has a representation

$$
h=\int_{S^{*}} K(\cdot, x) d \mu^{h}(x)
$$

for a Borel measure $\mu^{h}$ on $S^{*}$. This correspondence is one-one onto if $\mu^{h}$ assigns no mass to the set of "nonextreme" boundary points. We shall write $h=P I\left(d \mu^{h}\right)$.

3. Regular points. The first theorem is well known, but we give a proof for lack of a reference. 
THEOREM 1. If $p$ is in $S^{*}$, then these three statements are equivalent:

(1) If $h \geqq 0$ is any $\pi$-integrable harmonic function whose representing measure is absolutely continuous $[\mu]$ with density $f$ continuous at $p$, then $h$ converges to the "boundary value" $f(p)$ at $p$.

(2) If $h \geqq 0$ is any $\pi$-integrable harmonic function whose representing measure is absolutely continuous $[\mu]$ with a continuous density $f$, then $h$ converges to the "boundary value" $f(p)$ at $p$.

(3) If $U$ is any open neighborhood of $p$, then

$$
\lim _{j \rightarrow p} \operatorname{Pr}_{j}\left[x_{v} \in S^{*}-U\right]=0 .
$$

Proof. (1) $\Rightarrow(2)$. A fortiori.

$(2) \Rightarrow(3)$. Let $\rho(x, p) \geqq c>0$ for $x$ in $S^{*}-U$, and put $h=c^{-1} \int K(\cdot, x) \rho(x, p) d \mu(x)$. Then as $j \rightarrow p$,

$$
\begin{aligned}
0 & \leqq \operatorname{Pr}_{j}\left[x_{v} \in S^{*}-U\right]=\int_{S^{*}-U} K(j, x) d \mu(x) \\
& \leqq c^{-1} \int_{S^{*}-U} K(j, x) \rho(x, p) d \mu(x) \leqq h_{j} \rightarrow 0 .
\end{aligned}
$$

(3) $\Rightarrow(1)$. If $h=P I(f d \mu)$ and if $\epsilon>0$ is given, choose $U$ small enough so that $|f(x)-f(p)| \leqq \epsilon$ on $U$. Then

$$
\begin{aligned}
|h(j)-f(p)| & \leqq \int_{S^{*}} K(j, x)|f(x)-f(p)| d \mu(x)=\int_{U}+\int_{S^{*}-U} \\
& \leqq \epsilon+2\|f\| \operatorname{Pr}_{j}\left[x_{v} \in S^{*}-U\right] .
\end{aligned}
$$

For $j$ in a sufficiently small neighborhood of $p$, the last term is $<\epsilon$ by (3).

We take the equivalent conditions (1), (2), and (3) as a definition of regular point.

THEOREM 2. The set of regular points is a Borel set.

Proof. Fix a real-valued continuous function $f$ on $S^{*}$, and let $h=P I(f d \mu)$. Define $h^{*}$ and $h_{*}$ to be functions on $S^{*}-S$ with

$$
h^{*}(x)=\limsup _{j \rightarrow x, j \in S} h(j) \text { and } h_{*}(x)=\liminf _{j \rightarrow x, j \in S} h(j) .
$$

Then $h^{*}$ and $h_{*}$ are semicontinuous and hence Borel measurable. The set $R_{f}$ of points $p$ in $S^{*}$ where $h(j) \rightarrow f(p)$ as $j \rightarrow p$ is the union of some (necessarily countable) subset of $S$ and the set of those $p$ in $S^{*}-S$ at which the three measurable functions $f, h^{*}$, and $h_{*}$ are equal. Hence $R_{f}$ is a Borel set. 
Now choose a countable dense set of continuous functions on $S^{*}$, and let $R$ be the intersection of the corresponding sets $R_{f}$. $R$ is a Borel set and we claim it is just the set of regular points. In fact, $R$ contains the regular points by (2). In the reverse direction let $p$ be in $R$, let $f$ be continuous on $S^{*}$, and let $h=P I(f d \mu)$. Choose a sequence $\left\{f_{n}\right\}$ from the countable dense set converging uniformly to $f$, and let $h_{n}=P I\left(f_{n} d \mu\right)$. Then $\left\{h_{n}\right\}$ converges uniformly to $h$, and a $3 \epsilon$ argument shows that $\lim _{j \rightarrow p} h(j)=f(p)$. Hence $p$ is regular.

If harmonic measure $\mu$ assigns all its weight to one point, then that point is a regular point and no other point of $S^{*}$ is regular. Thus in this case it is trivial that almost every point is regular. On the other hand, it is easy to give an example of a chain in which the set of regular points is empty; practically any finite transient chain will do. Yet such a chain is an unfair example because regular points are interesting only when harmonic measure is completely on the boundary, as it is in the classical theory. For Markov chains this is the situation in which $P$ is stochastic, i.e., $P$ has row sums equal to one.

THEOREM 3. There exists a stochastic transient chain in which all pairs of states communicate, every one-point set of $S$ is open in $S^{*}$, the boundary contains just two points (both of them extreme), and the set of regular points is empty.

Proof. Let the state space consist of one full set of integers (written without prime marks) together with one set of integers with zero omitted (written with primes). The reader is asked to visualize the states arranged in the form of a cross $X$ with infinite horizontal rays extending from each of the four points of the $X$. Along either primed ray the process marches deterministically one step at a time toward 0 , and from $1^{\prime}$ and $(-1)^{\prime}$ it moves to 0 on the next step. From state 0 it moves to states 1 and -1 with probabilities $p_{1}$ and $p_{-1}$, respectively, and it stays at 0 with probability $1-p_{1}-p_{-1}$. From any state on the unprimed rays it moves with probability $p_{i}$ to the next unprimed state $i$ that is farther away from 0 ; also from the given state the process moves with probability $q_{i}=1-p_{i}$ to the same numbered state on the unprimed rays (either $(i+1)^{\prime}$ or $\left.(i-1)^{\prime}\right)$.

All pairs of states communicate if the $p$ 's and $q$ 's are chosen to be nonvanishing, and we can take the starting vector $\pi$ to be a unit mass at 0 . If $i$ is on the unprimed axis, we define $\beta_{i}$ by

$$
\beta_{0}=1, \quad \beta_{i}=p_{1} p_{2} \cdots p_{i} \text { if } i>0, \quad \beta_{i}=p_{-1} p_{-2} \cdots p_{i} \text { if } i<0 .
$$

Put 


$$
\beta_{\infty}=\lim _{i \rightarrow+\infty} \beta_{i} \text { and } \beta_{-\infty}=\lim _{i \rightarrow-\infty} \beta_{i} .
$$

The process is transient if either one of $\beta_{\infty}$ and $\beta_{-\infty}$ is strictly positive. We shall assume that the $p$ 's are chosen so that both $\beta_{\infty}$ and $\beta_{-\infty}$ are strictly positive.

We claim the sequences $\left\{1,1^{\prime}, 2,2^{\prime}, 3, \cdots\right\}$ and $\left\{-1,(-1)^{\prime}\right.$, $\left.-2,(-2)^{\prime}, \ldots\right\}$ are Cauchy. By symmetry it suffices to show that the first sequence is Cauchy. If $H_{i j}$ denotes the hitting probability from $i$ to $j$, we have

$$
\begin{aligned}
& K(0, j)=1, \quad j \text { arbitrary (primed or unprimed) } \\
& K(i, j)=\frac{N(i, j)}{N(0, j)}=\frac{H_{i j}}{H_{0 j}}=\frac{H_{i j}}{H_{0 i} H_{i j}}=\frac{1}{H_{0 i}}, \quad\left\{\begin{array}{l}
i \text { unprimed }>0 \\
j \text { arbitrary beyond } i
\end{array}\right. \\
& K(i, j)=\frac{H_{i j}}{H_{0 j}}=\frac{H_{i 0} H_{0 j}}{H_{0 j}}=H_{i 0}, \quad\left\{\begin{array}{l}
i \text { unprimed }<0 \\
j \text { arbitrary }>0
\end{array}\right. \\
& K(i, j)=\frac{H_{i 0} H_{0 j}}{H_{0 j}}=H_{i 0}=1, \quad\left\{\begin{array}{l}
i \text { primed } \\
j \text { farther from } 0 \text { than } i .
\end{array}\right.
\end{aligned}
$$

Thus the first sequence is Cauchy.

The fact that two Cauchy sequences exhaust $S$ except for finitely many states implies that the limits of these sequences in $S^{*}$ are the only limit points in $S^{*}$. If we denote the limits by $+\infty$ and $-\infty$, we have

$$
\begin{aligned}
& K(i,+\infty)= \begin{cases}1 / H_{0 i} & \text { if } i \text { is unprimed and } \geqq 0 \\
H_{i 0} & \text { if } i \text { is unprimed and }<0 \\
1 & \text { if } i \text { is primed }\end{cases} \\
& K(i,-\infty)= \begin{cases}1 / H_{0 i} & \text { if } i \text { is unprimed and } \leqq 0 \\
H_{i 0} & \text { if } i \text { is unprimed and }>0 \\
1 & \text { if } i \text { is primed. }\end{cases}
\end{aligned}
$$

It is readily verified that

$$
\begin{aligned}
H_{i 0} & = \begin{cases}\left(\beta_{i}-\beta_{\infty}\right) / \beta_{i} & \text { if } i \text { is unprimed and }>0 \\
\left(\beta_{i}-\beta_{-\infty}\right) / \beta_{i} & \text { if } i \text { is unprimed and }<0\end{cases} \\
H_{0 i} & = \begin{cases}\beta_{i} /\left(\beta_{i}+\beta_{-\infty}\right) & \text { if } i \text { is unprimed and }>0 \\
\beta_{i} /\left(\beta_{i}+\beta_{\infty}\right) & \text { if } i \text { is unprimed and }<0 .\end{cases}
\end{aligned}
$$

From these equations we see that $K(\cdot,+\infty)$ and $K(\cdot,-\infty)$ are harmonic and linearly independent. It follows that $+\infty$ and $-\infty$ are 
extreme boundary points and that every one-point set of $S$ is open. Harmonic measure $\mu$ satisfies

$$
\mu(+\infty)=\frac{\beta_{\infty}}{\beta_{\infty}+\beta_{-\infty}}>0 \text { and } \mu(-\infty)=\frac{\beta_{-\infty}}{\beta_{\infty}+\beta_{-\infty}}>0 .
$$

It is trivial that no point of $S$ is a regular point. To see that $+\infty$ is not regular, let $U$ be any neighborhood of $+\infty$ which does not contain $-\infty$. Then

$$
\operatorname{Pr}_{i}\left[x_{\mathfrak{v}} \in S^{*}-U\right]=\int_{S^{*}-U} K(i, x) d \mu(x)=K(i,-\infty) \mu(-\infty) .
$$

As $i$ tends to $+\infty$ through primed states, the right side is bounded away from zero. Hence $+\infty$ is not regular. Similarly $-\infty$ is not regular.

\section{REFERENCES}

1. J. L. Doob, Discrete potential theory and boundaries, J. Math. Mech. 8 (1959) 433-458.

2. G. C. Evans, Potentials of positive mass. II, Trans. Amer. Math. Soc. 38 (1935), 201-236.

3. G. A. Hunt, Markoff chains and Martin boundaries, Illinois J. Math. 4 (1960), $313-340$.

4. O. D. Kellogg, Foundations of potential theory, Dover, New York, 1929.

5. J. G. Kemeny, J. L. Snell and A. W. Knapp, Denumerable Markov chains, Van Nostrand, Princeton, N. J., 1966.

6. R. S. Martin, Minimal positive harmonic functions, Trans. Amer. Math. Soc. 49 (1941), 137-172.

Massachusetts Institute of Technology 